\title{
Caracterização da violência obstétrica na produção científica: uma revisão integrativa
}

\author{
Characterization of obstetric violence in scientific production: an integrative review \\ Caracterización de la violencia obstétrica en la producción científica: una revisión \\ integradora
}

Karine Gondin Ribeiro ${ }^{1}$, Tatiane Cristina Dos Santos Michelini Ribeiro', Rosiane dos Santos Ferreira Dias $^{1}$, Maria Esméria Neta ${ }^{1}$, Maria Geralda Leite ${ }^{1}$, Keila Santos Silva ${ }^{1}$, Daniel Silva Moraes ${ }^{2}$, Ricardo Otávio Maia Gusmão², Maria Alice de Freitas $^{3}$, Renê Ferreira da Silva Junior ${ }^{3 *}$.

\section{RESUMO}

Objetivo: Descrever como é caracterizada a violência obstétrica na produção científica nos últimos 12 anos. Métodos: Trata-se de um estudo de revisão integrativa da literatura no qual foram utilizadas as bases de dados LILACS, Scielo e PUBMED para a busca e seleção de artigos em um corte temporal de 12 anos. Resultados: Após leitura e análise dos artigos considerados elegíveis foram definidas as seguintes categorias de resposta que guiaram a análise dos dados coletados: "Procedimentos desnecessários e sem consentimento"; "Intervencionismo e maus tratos durante o parto"; "Abuso físico, emocional e sexual por parte do parceiro íntimo". Um dos autores pesquisados detalhou a violência obstétrica descrevendo-a como: "cuidado não consentido, direito à informação; cuidado não confidencial; abuso verbal; abandono do cuidado; cuidado discriminatório; e abuso físico", sendo assim definida a categoria "supressão de direitos e de cuidados". Considerações finais: A análise dos artigos consultados aponta para um conceito de violência obstétrica relacionado a realização de procedimentos sem o consentimento da gestante em qualquer situação onde não há previsão legal para sustentar a execução da ação de saúde.

Palavras-chave: Violência contra a mulher, Parto humanizado, Revisão.

\begin{abstract}
Objective: To describe how obstetric violence is characterized in scientific production in the last 12 years. Methods: This is an integrative literature review study in which the LILACS, Scielo and PUBMED databases were used for the search and selection of articles in a 12-year period. Results: After reading and analyzing the articles considered eligible, the following response categories were defined, which guided the analysis of the data collected: "Unnecessary procedures and without consent"; "Interventionism and mistreatment during childbirth"; "Physical, emotional and sexual abuse by the intimate partner". One of the researched authors detailed obstetric violence, describing it as: "care not allowed, right to information; non-confidential care; verbal abuse; abandoning care; discriminatory care; and physical abuse", thus defining the category "suppression of rights and care". Final considerations: The analysis of the articles consulted points to a concept of obstetric violence related to the performance of procedures without the consent of the pregnant woman in any situation where there is no legal provision to support the execution of the health action.
\end{abstract}

Key words: Violence against women, Humanizing delivery, Review.

\section{RESUMEN}

Objetivo: Describir cómo se caracteriza la violencia obstétrica en la producción científica en los últimos 12 años. Métodos: Se trata de un estudio de revisión integradora de la literatura en el que se utilizaron las bases de datos LILACS, Scielo y PUBMED para la búsqueda y selección de artículos en un período de 12 años.

\footnotetext{
${ }^{1}$ Faculdades Unidas do Norte de Minas Gerais (FUNORTE), Montes Claros - MG.

2 Universidade Estadual de Montes Claros (UNIMONTES), Montes Claros - MG.

${ }^{3}$ Instituto Federal de Educação, Ciência e Tecnologia de Santa Catarina (IFSC), Joinville - SC.

*E-mail: rene.junior@ifsc.edu.br
} 
Resultados: Luego de la lectura y análisis de los artículos considerados elegibles, se definieron las siguientes categorías de respuesta, que guiaron el análisis de los datos recolectados: "Procedimientos innecesarios y sin consentimiento"; "Intervencionismo y maltrato durante el parto"; "Abuso físico, emocional y sexual por parte de la pareja íntima". Una de las autoras investigadas detalló la violencia obstétrica, describiéndola como: "atención no permitida, derecho a la información; atención no confidencial; abuso verbal; abandono del cuidado; atención discriminatoria; y maltrato físico", definiendo así la categoría "supresión de derechos y cuidados". Consideraciones finales: El análisis de los artículos consultados apunta a un concepto de violencia obstétrica relacionado con la realización de procedimientos sin el consentimiento de la gestante en cualquier situación donde no exista una disposición legal que sustente la ejecución de la acción de salud.

Palabras clave: Violencia contra la mujer, Parto humanizado, Revisión.

\section{INTRODUÇÃO}

A humanização do parto no Brasil cresceu e se consolidou nas últimas décadas expandindo para vários estados. Nos anos 70 teve início uma ampliação do movimento por meio da aproximação de profissionais com práticas tradicionais e populares e também com conceitos paralelos e alternativos, tais como a inserção da mulher e do bebê como centro da assistência (BOURGUIGNON AN e GRISOTTI M, 2020).

Nessa discussão, a definição de disrespect and abuse during childbirth tem sido internacionalmente usado para denominar o que no cenário brasileiro é conhecido como violência obstétrica, violência durante o parto, violência estrutural ou ainda institucional na assistência ao parto. Esta nomenclatura foi sugerida para identificar qualquer ação de violência dirigido à mulher gestante, parturiente ou puérpera ou ao seu filho, perpetuado durante a atenção profissional, que denote desrespeito a sua autonomia, integridade física e mental, desejos, preferenciais e sentimentos. A violência obstétrica foi recentemente identificada pela Organização Mundial de Saúde, em 2014, como uma questão de saúde pública que atinge de forma direta as mulheres e seus bebês (DINIZ SG, et al., 2015; OMS, 2014).

A conceituação do termo violência obstétrica pode ser difícil tendo em vista que não é claramente especificado nos dispositivos legais ou na produção científica. Assim, a ausência de clareza conceitual impede a tipificação penal e, portanto, impossibilita ações mais pontuais em termos de classificação, diagnóstico e abordagem de casos. De acordo com dados do Ministério da Saúde, o número de partos hospitalares representa mais de $98 \%$ do total de partos realizados no Brasil e o incremento entre 2007 e 2011 foi significativo, passando de $46,5 \%$ para $53,88 \%$ o total de cesarianas com importantes variações entre o serviço público e privado (ZANARDO GLP, et al., 2017).

Esse contexto é considerado preocupante quando se avalia as recomendações dos órgãos internacionais, como a Organização Mundial de Saúde, pois a recomendação é de que a taxa de partos cesarianos estejam entre 10 a $15 \%$ do total de partos realizados. Tal indicação está balizada em pesquisas que indicam que uma taxa maior que $15 \%$ não acarreta diminuição nas mortes maternas e igualmente não trazem melhores desfechos de saúde para o binômio mãe-filho (BRASIL, 2014; BRASIL, 2015).

A grande quantidade de intervenções e o processo de medicalização durante o parto resultam na retirada do protagonismo da mulher, impossibilitando-a ter autonomia e capacidade de decidir de forma livre acerca do seu próprio corpo (MUNIZ BMV e BARBOSA RM, 2012). A Organização Mundial de Saúde afirma que toda mulher possui o direito a uma atenção à saúde que garanta dignidade e respeito no transcorrer de toda a experiência do parto, independentemente de qualquer característica da mulher, seja classe social, nível de escolaridade, dentre outros. Qualquer ação que seja desrespeitosa, abuso ou maus tratos equivale a violação dos direitos fundamentais das mulheres (OMS, 2014).

É considerada frequente a violência obstétrica e institucional e por isso constitui área de grande relevância pública no cenário brasileiro. Apesar dessa ocorrência importante assim como a violência doméstica e sexual, a violência institucionalizada ficou oculta por muito tempo. Importante ainda ressaltar que a violência é também considerada de gênero, tendo em vista que se caracteriza pela especificidade contra a mulher e por causa da condição de vulnerabilidade (BRASIL, 2014). 
A falta de organização, estrutura adequada e capacitação deficitária assim como a falta de uma prática baseada em evidências pode intensificar a violência institucional. Gestores, profissionais e a sociedade precisam ser informados e motivados ao reconhecimento da violência obstétrica como um ato frequente e que deve ser abolido definitivamente da prática de saúde (GUIMARÃES LBE, et al., 2018).

As dificuldades conceituais e de definição da violência institucional contra a mulher são uma realidade no Brasil, as pactuações atuais e a necessidade de consolidação dos princípios do Sistema Único de Saúde (SUS) na área obstétrica incluem estratégias de humanização, acolhimento e reordenação da assistência com ênfase nos direitos da mulher. Tendo em vista este contexto, o presente estudo teve como objetivo descrever como é caracterizada a violência obstétrica na produção científica nos últimos 12 anos.

\section{MÉTODOS}

Trata-se de um estudo de revisão integrativa da literatura. A revisão integrativa é uma estratégia metodológica de pesquisa que constitui ferramenta importante, pois possibilita a análise de subsídios na literatura de maneira mais intensa e sistemática, além de divulgar informações cientificas resultado de estudos de outros autores. Este método permite um resumo do conhecimento já criado e possibilita bases para o progresso da atenção à saúde. Oferece ainda aos profissionais da área da saúde o acesso de forma mais rápida aos resultados importantes de estudos que se baseiam nas condutas ou a tomada de decisão, resultando em um conhecimento crítico (MARCONI MA e LAKATOS EM, 2017).

Para a realização da revisão integrativa foram seguidas seis fases interdependentes: 1) elaboração da pergunta norteadora, 2) busca ou amostragem na literatura, 3) coleta de dados, 4) análise crítica dos estudos incluídos, 5) discussão dos resultados e 6) apresentação da revisão integrativa (SOUZA MT, et al, 2010). Considerou-se como questão norteadora: Como é caracterizada a violência obstétrica na produção científica nos últimos 12 anos?

Foram utilizadas as bases de dados Literatura Latino-Americana e do Caribe em Ciências da Saúde (LILACS), Scientific Electronic Library Online (Scielo) e Medical Literature Analysis and Retrieval System Online (MEDLINE) para a busca e seleção de artigos. Foram utilizados os descritores "violência contra a mulher e parto humanizado" (disponíveis nos Descritores em Ciências em Saúde - DeCS) na busca em bases regionais, para a busca na PUBMED foram utilizados os descritores (disponíveis no Medical Subject Headings - MESH) "violence" e "obstetric". Foi utilizado o operador boleano "and" para os cruzamentos realizados (Quadro 1).

Após a busca inicial com os descritores e utilização dos critérios de inclusão (artigo publicado nos últimos 12 anos, artigo em português, inglês ou espanhol e artigo com temática específica) foi realizada a leitura dos resumos e títulos para seleção inicial dos textos. Em seguida, procedeu-se à leitura na íntegra dos artigos, seleção de trechos específicos para resposta à questão de pesquisa e realizada busca de palavras-chave no artigo para definição das categorias de resposta.

Para a extração dos dados foi utilizado instrumento validado por Ursi ES (2005) com os seguintes itens: dados de identificação do artigo (título, autores, nome do periódico, ano de publicação, volume e número), tipo de estudo, local de estudo, objetivo do estudo, eixo temático, classificação Qualis/Capes, periódico de publicação, nível de evidência, desfechos, dentre outros. A seleção dos artigos foi realizada de forma independente pelos pesquisadores, as divergências foram solucionadas com base no instrumento validado.

A partir da seleção dos artigos foi construído um quadro com dados sobre os artigos elegíveis definido como quadro sinóptico (Quadro 1). A categorização das respostas obedeceu aos critérios pré-estabelecidos para resposta à questão de pesquisa que foram: conceituação de violência obstétrica, definição do termo de forma prática e exemplificação da violência obstétrica. Para os artigos de base qualitativa utilizou-se a definição dos sujeitos para a construção conceitual da categoria. Foram excluídas teses, dissertações, cartilhas, trabalhos de conclusão de curso e monografias, além de artigos que não abordassem de forma relevante a temática do estudo. 


\section{RESULTADOS E DISCUSSÃO}

Na busca inicial nas bases de dados secundários utilizando os descritores "violência contra a mulher e parto humanizado na Scielo foram encontrados 11 artigos elegíveis, sendo excluídos oito artigos devido a não atenderem os critérios de inclusão estabelecidos, na LILACS foram encontrados inicialmente 6 artigos, sendo selecionados dois deles após a análise minuciosa dos manuscritos. Já na base de dados PUBMED com os descritores "violence" e "obstetric" (utilizando-se o operador boleano "and") foram encontrados 249 artigos, sendo selecionados três artigos por atenderem de forma inequívoca aos critérios de elegibilidade. Sendo assim, a amostra final foi de 8 artigos (Quadro 1).

Quadro 1 - Quadro sinóptico com os resultados dos artigos considerados elegíveis para resposta à questão de pesquisa "Como é caracterizada a violência obstétrica pelos autores nos últimos 12 anos?".

\begin{tabular}{|c|c|c|c|c|}
\hline Artigos & Autor/ano & Metodologia & $\begin{array}{l}\text { Base de } \\
\text { dados }\end{array}$ & $\begin{array}{l}\text { Resposta à questão } \\
\text { norteadora do estudo }\end{array}$ \\
\hline $\begin{array}{c}\text { Percepção de } \\
\text { enfermeiras obstétricas } \\
\text { acerca da violência } \\
\text { Obstétrica } \\
\end{array}$ & $\begin{array}{l}\text { LEAL SYP, } \\
\text { et al., } 2018\end{array}$ & $\begin{array}{l}\text { Estudo } \\
\text { exploratório e } \\
\text { qualitativo }\end{array}$ & LILACS & $\begin{array}{l}\text { "Procedimentos } \\
\text { desnecessários e sem } \\
\text { consentimento" }\end{array}$ \\
\hline $\begin{array}{c}\text { Violência obstétrica no } \\
\text { processo de parturição } \\
\text { em maternidades } \\
\text { vinculadas à Rede } \\
\text { Cegonha }\end{array}$ & $\begin{array}{l}\text { RODRIGUE } \\
\text { S FAC, et al., } \\
2017\end{array}$ & $\begin{array}{c}\text { Estudo } \\
\text { descritivo } \\
\text { quantitativo }\end{array}$ & LILACS & $\begin{array}{l}\text { "Procedimentos } \\
\text { desnecessários e sem } \\
\text { consentimento" }\end{array}$ \\
\hline $\begin{array}{l}\text { Violência obstétrica na } \\
\text { visão de enfermeiras } \\
\text { obstetras }\end{array}$ & $\begin{array}{l}\text { SILVA MG, } \\
\text { et al., } 2014\end{array}$ & $\begin{array}{l}\text { Estudo baseia- } \\
\text { se em um relato } \\
\text { de experiência }\end{array}$ & LILACS & $\begin{array}{c}\text { "Procedimentos } \\
\text { desnecessários e sem } \\
\text { consentimento" }\end{array}$ \\
\hline $\begin{array}{l}\text { O discurso da violência } \\
\text { obstétrica na voz das } \\
\text { mulheres e dos } \\
\text { profissionais de saúde }\end{array}$ & $\begin{array}{l}\text { OLIVEIRA } \\
\text { VJ, PENNA } \\
\text { CMM., } 2017\end{array}$ & $\begin{array}{l}\text { Trata-se de um } \\
\text { estudo } \\
\text { interpretativo, } \\
\text { com abordagem } \\
\text { qualitativa } \\
\end{array}$ & SCIELO & $\begin{array}{l}\text { "Intervencionismo e maus } \\
\text { tratos durante o parto" }\end{array}$ \\
\hline $\begin{array}{l}\text { Intimate partner violence } \\
\text { against pregnant women: } \\
\text { study about the } \\
\text { repercussions on the } \\
\text { obstetric and neonatal } \\
\text { results }\end{array}$ & $\begin{array}{l}\text { RODRIGUE } \\
\text { S DP, } 2014\end{array}$ & $\begin{array}{l}\text { Estudo analítico, } \\
\text { observacional e } \\
\text { descritivo }\end{array}$ & PUBMED & $\begin{array}{l}\text { "Abuso físico, emocional } \\
\text { e sexual por parte do } \\
\text { parceiro íntimo" }\end{array}$ \\
\hline $\begin{array}{c}\text { In person versus } \\
\text { Computer Screening for } \\
\text { Intimate Partner Violence } \\
\text { Among Pregnant Patients }\end{array}$ & $\begin{array}{l}\text { DADO D, et } \\
\text { al., } 2012\end{array}$ & $\begin{array}{l}\text { Estudo } \\
\text { observacional } \\
\text { qualitativo }\end{array}$ & PUBMED & $\begin{array}{l}\text { "Abuso físico, emocional } \\
\text { e sexual por parte do } \\
\text { parceiro íntimo" }\end{array}$ \\
\hline $\begin{array}{l}\text { Can a simulation-based } \\
\text { training program impact } \\
\text { the use of evidence } \\
\text { based routine practices at } \\
\text { birth? Results of a } \\
\text { hospital-based cluster } \\
\text { randomized trial in Mexico }\end{array}$ & $\begin{array}{l}\text { FRITZ J, et } \\
\text { al., } 2017\end{array}$ & $\begin{array}{l}\text { Ensaio clínico } \\
\text { randomizado } \\
\text { controlado }\end{array}$ & PUBMED & $\begin{array}{l}\text { "Procedimentos } \\
\text { desnecessários e sem } \\
\text { consentimento" }\end{array}$ \\
\hline $\begin{array}{l}\text { Women's experiences of } \\
\text { mistreatment during } \\
\text { childbirth: A comparative } \\
\text { view of home- and facility- } \\
\text { based births in Pakistan }\end{array}$ & $\begin{array}{l}\text { HAMEED W, } \\
\text { AVAN BI., } \\
2018\end{array}$ & $\begin{array}{c}\text { Coorte } \\
\text { prospectiva }\end{array}$ & PUBMED & $\begin{array}{l}\text { Supressão de direitos e } \\
\text { de cuidados }\end{array}$ \\
\hline
\end{tabular}

Fonte: Ribeiro KG, et al., 2021. 
Após leitura e análise dos artigos considerados elegíveis foram definidas as seguintes categorias de resposta que guiaram a análise dos dados coletados: Procedimentos desnecessários e sem consentimento; intervencionismo e maus tratos durante o parto; abuso físico, emocional e sexual por parte do parceiro íntimo.

No cenário nacional, a implantação do Programa de Humanização do Pré-Natal e Nascimento, conhecido também pela sigla PHPN, no ano de 2000 , teve como objetivo resolver as reivindicações sociais pela melhora da qualidade da atenção ao parto e nascimento, temática que tem sido enfoque de atenção em várias regiões do país. O propósito era o de possibilitar uma intensa discussão sobre o assunto (RODRIGUES DP, et al., 2015). Nessa discussão, a referida estratégia do programa é consequência de um movimento de caráter internacional que teve início há aproximadamente 30 anos contra o uso, considerado irracional e danoso, de tecnologias invasivas no transcorrer do parto, almejando priorizar a qualidade da relação mulher/usuária dos serviços de saúde e o trabalhador de saúde (AGUIAR JM e D'OLIVEIRA AFL, 2011; AGUIAR JM, et al., 2013).

Nos dias atuais o parto tem sido encarado como um processo de caráter patológico, que tem trazido o uso da tecnologia do parto dirigido, sendo que a parturiente se encontra, frequentemente, em posição semiimobilizada, com os membros inferiores abertos e em posição superior, com ingestão oral de líquidos e alimentos proibida, sujeita ao uso de fármacos para o processo de indução do parto e o uso frequente de episiotomia e eventual do fórceps. Este é o modelo de assistência ao parto vaginal mais habitual no cenário brasileiro, sendo realizado, geralmente, por um profissional médico em um serviço hospitalar, razão pela qual também é denominado como parto normal hospitalar (MEDEIROS NCM, et al., 2016).

A violência obstétrica é descrita de formas variadas principalmente ligada ao uso de procedimentos invasivos, tais como punção venosa e administração de ocitocina e o abuso emocional com a utilização de um discurso de intimidação com as mulheres. O profissional, imerso na prática, pode participar das situações e realizar os procedimentos sem consentimento da mulher e não perceber que se trata de um ato violento. Não apenas os profissionais podem ter dificuldade em perceber a situação de violência como as parturientes também, já que não tem o conhecimento necessário para discernir o fato. Apenas as mulheres que participam de grupos em que o conhecimento é difundido ou profissionais engajados com a causa podem ter uma reação avessa à violência identificando as situações de forma oportuna (LEAL SYP, et al., 2018).

A violência obstétrica é um fato de caráter socialmente complexo no campo da saúde da mulher. Sua prevenção exige transformações nas práticas de cuidado no transcorrer do desenvolvimento da fase gravídico-puerperal, almejando diminuir intervenções médicas inapropriadas e sem necessidade, que assim, podem acarretar prejuízos à saúde emocional e também física das mulheres (CARLOS GA, et al., 2019).

O tratamento de caráter abusivo no transcorrer do desenvolvimento do trabalho de parto e parto fere os direitos humanos, sendo possível afetar de maneira negativa os resultados dessas experiências e impedir que a mulher no futuro busque por assistência. Ademais, é encarada como uma violência de cunho estrutural que as torna susceptíveis ao sofrimento e à morte (MARRERO L e BRÜGGEMANN OM, 2018).

Uma das situações citadas nos estudos é o jejum durante o trabalho de parto, tal procedimento pode trazer o desconforto perfeitamente evitável da hipoglicemia, tendo em vista o gasto energético e o consumo de glicose da parturiente. Sendo assim, não há uma justificativa considerável no fato de preconizar o jejum durante o trabalho de parto normal. Os profissionais, no cotidiano do trabalho, podem executar ações que julgam não ser um ato violento por não serem atos de comissão (como o caso do jejum obrigatório) ou ainda executarem atos com a intenção de acompanhamento rigoroso e monitoramento como o excesso de toques vaginais em um curto espaço de tempo provocando edemas vaginais e desconfortos (RODRIGUES FAC, et al., 2017).

O uso de ocitocina e a falta de suporte emocional, as ordens para parar de gritar, apelidos e as manobras podem ser as situações que mais frequentemente definem os atos violentos na sala de parto. Assim, observase a importância de profissionais capacitados e aptos para proporcionar um parto humanizado disseminando segurança e apoio para as mulheres (RODRIGUES FAC, et al., 2017).

Assim, compreende-se que há necessidade de mudanças no cenário brasileiro em relação a violência obstétrica, observa-se despreparo, negligência e imperícia na prática de profissionais da obstetrícia, sejam 
eles médicos, enfermeiros, enfermeiros obstetras, médicos obstetras ou auxiliares de enfermagem. A violência não está ligada apenas ao ato do profissional mas começa com a inobservância da ambiência, com a ausência do acolhimento ou da assistência sem profissionais capacitados. Faz-se necessário então, para uma assistência obstétrica diferenciada, o investimento constante na formação dos profissionais (SILVA MG, et al., 2014).

Outro ponto de análise foi a violência causada por parceiro íntimo (VPI), sendo definida como o comportamento dentro de uma relação íntima que acarreta danos físicos, psicossociais ou sexuais, o que inclui ações de agressão física, coerção de cunho sexual, abuso de esfera psicológica e ações intimidadoras (OMS, 2012). Essa forma de violência deve ser reconhecida pela equipe multiprofissional, sendo que tal percepção leva a um acolhimento e uma abordagem mais significativa. São fundamentais as ações que produzem uma detecção precoce de casos de violência perpetrada pelo parceiro íntimo, tendo em vista os riscos associados à situação (RODRIGUES DP, et al., 2014)

No que tange às repercussões obstétricas e para o neonato, os efeitos da VPI podem ser importantes, entretanto ainda não se percebe um consenso quanto ao grau dessa repercussão ou, de forma oposta, se 0 fenômeno da gravidez leva a redução das situações de violência por parte do parceiro (RODRIGUES DP, et al., 2014).

Dado D, et al. (2012) também cita a violência contra a mulher causada pelo parceiro íntimo sugerindo uma abordagem realizada de forma sistemática; os autores fizeram um estudo com mulheres na primeira consulta obstétrica (pré-natal) considerando que este seria o momento de um contato maior quanto ao tempo de entrevista e, portanto, mais oportuno para a investigação em função da frequência do uso deste momento para o conhecimento da violência contra a mulher.

Em um estudo realizado com o intuito de avaliar o impacto de um programa para treinamento de equipes para a assistência (baseada em evidências científicas) os autores referem-se à violência obstétrica de forma secundária citando a necessidade de remodelar os esforços em todos os países para um cuidado mais eficiente e eficaz (FRITZ J, et al, 2017).

É bem abrangente a conceituação de Hammed W e Avan B (2018) referindo-se ao termo violência obstétrica como cuidado não consentido, supressão do direito à informação; cuidado não confidencial; abuso verbal; abandono do cuidado; cuidado discriminatório e abuso físico. Parte considerável das mulheres que procuram a atenção obstétrica, principalmente em países periféricos, encontra-se em situação de risco de violência por parte da equipe que as assiste.

Segundo a Organização Mundial de Saúde, os abusos e ausência de respeito durante o parto e trabalho de parto em serviços de saúde ocorrem de maneiras diversas, tais como: abusos de caráter verbal e humilhações intensas (em muitos casos associadas ao machismo); violência de caráter físico, como por exemplo, a conhecida manobra de Kristeller (pressão no útero para a expulsão do bebê) e o não consentimento das mulheres antes da realização dos diversos procedimentos (OMS, 2014).

Pode-se citar ainda procedimentos médicos de aspecto coercitivo ou não autorizados pela mulher, ausência de privacidade no ambiente de internação, recusa de internação nos serviços de saúde, negativa em administrar analgesia, assistência negligente no transcorrer do parto e trabalho de parto que pode acarretar desfechos desfavoráveis evitáveis; detenção da parturiente nos serviços de saúde, em seguida ao parto, em razão da incapacidade de pagamento, administração de ocitocina sintética e o impedimento de acompanhamento durante o parto (OMS, 2014).

Urge, portanto, que os cuidados sejam centralizados na mulher e que o serviço reordene sua prática incluindo estratégias de sensibilização de profissionais e ainda, que oriente o cuidado de saúde no nível interpessoal ao passo que observa o indivíduo de forma holística (HAMMED W e AVAN B, 2018).

A Organização Mundial de Saúde ressalta que o parto é um processo natural que não exige controle e sim cuidados assistenciais. Baseando-se nesta compreensão esta entidade sugere uma participação mais intensa do enfermeiro obstetra na assistência ao processo de parto e parto, baseando na perspectiva de que a formação deste profissional é direcionada para o cuidado e não para a intervenção (OMS, 2014). 
Em estudo realizado por Oliveira VJ e Penna CMM (2017) percebeu-se que os enfermeiros obstetras sentem-se desmotivados na sala de parto em função do ambiente e da forma como a parturiente é tratada, principalmente no momento expulsivo, existem casos em que ocorrem gritos e ofensas. No mesmo estudo a equipe médica citava o termo violência obstétrica reduzindo seu impacto ao referir que atualmente "tudo é violência" e que o uso exacerbado do termo pela mídia reduz a autonomia do obstetra.

A formação dos profissionais enfermeiros acerca da violência obstétrica deve ser mais abrangente, uma vez que estes profissionais são coadjuvantes dessas experiências, e nelas exercem relevante função ao possibilitar a qualidade de assistência de saúde que as mulheres precisam e são asseguradas em ter, enquanto cidadãs de direito. Salienta-se que, quando a mulher é educada de forma adequada, possui menos possibilidade de ser vítima de violência obstétrica. Dessa forma, o papel do profissional em formação é essencial em relação a violência obstétrica, pois, este profissional pode diminuir as taxas desse agravo e transformar a realidade social (SILVA TM, et al., 2020).

Considera-se assim, que a proposição de ações de prevenção e confrontação da violência obstétrica envolve a formação acadêmica, conscientização das gestantes, enfrentamento da sociedade e elaboração de leis e políticas públicas, dentre outras ações (JARDIM DMB e MODENA CM, 2018).

\section{CONSIDERAÇÕES FINAIS}

A análise dos artigos consultados aponta para um conceito de violência obstétrica relacionado a realização de procedimentos sem o consentimento da gestante em qualquer situação em que não há previsão legal para sustentar a execução da ação de saúde. Tal fato é relevante e corrobora a tese de que a supressão da autonomia da mulher na situação de vulnerabilidade do evento do parto predispõe a mais riscos associados. Outro conceito relevante citado pelos autores como definição de violência obstétrica diz respeito ao intervencionismo sem um resultado efetivo e baseado em questões empíricas. Importante ressaltar a necessidade de se ampliar a produção científica nacional nesta temática tendo em vista seu impacto social e individual.

\section{REFERÊNCIAS}

1. AGUIAR JM, D'OLIVEIRA AFL. Violência institucional em maternidades públicas sob a ótica das usuárias. Interface comun saúde educ, 2011;15(36): 79-91.

2. AGUIAR JM, et al. Violência institucional, autoridade médica e poder nas maternidades sob a ótica dos profissionais de saúde. Cad saúde pública. 2013; 29(11): 2287-2296.

3. BRASIL. Ministério da Saúde. Humanização do parto e do Nascimento. Brasília: Ministério da Saúde, 2014; 465p.

4. BRASIL. Ministério da Saúde. Cadernos HumanizaSUS - Volume 4: Humanização do parto e do nascimento. Brasília, DF: UECE/ Ministério da Saúde, 2014.

5. BRASIL. Ministério da Saúde. Diretrizes de Atenção à Gestante: a operação cesariana. Comissão Nacional de Incorporação de Tecnologias no SUS (CONITEC). Brasília: Ministério da Saúde, 2015.

6. BOURGUIGNON AN, GRISOTT M. A humanização do parto e nascimento no Brasil nas trajetórias de suas pesquisadoras. História, Ciências, Saúde - Manguinhos. 2020; 27(2): 485-502

7. CARLOS GA, et al. Profile of the participants of an advanced course in obstetric nursing. Rev Min Enferm, 2019;23 (1): $1-7$

8. DADO D. In person versus computer screening for intimate partner violence among pregnant patients. Patient Educ Couns, 2012; 88(3): 443-448.

9. DINIZ SG, et al. Violência obstétrica como questão para a saúde pública no Brasil: origens, definições, tipologia, impactos sobre a saúde materna, e propostas para sua prevenção. Journal of Human Growth and Development, $2015 ; 25(3): 1-8$.

10. FRITZ J, et al. Can a simulation-based training program impact the use of evidence based routine practices at birth? Results of a hospital-based cluster randomized trial in Mexico. Plos One, 2017; 12(3): 1-15.

11. GUIMARÃES LBE, et al. Violência obstétrica em maternidades públicas do estado do Tocantins. Estudos Feministas, 2018; 26(1):1-11.

12. HAMEED W, AVAN BI. Women's experiences of mistreatment during childbirth: A comparative view of home- and facility-based births in Pakistan. Plos One, 2018; 13(3): 1-17.

13. JARDIM DMB, MODENA CM. A violência obstétrica no cotidiano assistencial e suas características. Rev. Latino-Am. Enfermagem. 2018; 26(1): 1-12. 
14. LEAL SYP, et al. Percepção de enfermeiras obstétricas acerca da violência obstétrica. Cogitare Enferm, 2018; 23(2):1-7.

15. MARCONI MA, LAKATOS EM. Metodologia do trabalho científico. 8 ed. São Paulo: Atlas, 2017; 242p.

16. MEDEIROS NCM, et al. Violência obstétrica: percepções acerca do parto normal. Temas em saúde, 2016; 16(3): 503508.

17. MARRERO L, RÜGGEMANN OM. Institutional violence during the parturition process in Brazil: integrative review. Rev Bras Enferm, 2018;71(3):1152-1161.

18. MUNIZ BMV, BARBOSA RM. Problematizando o atendimento ao parto: cuidado ou violência? In: Memorias convención internacional de salud pública. Memorias Convención Internacional de Salud Pública. Cuba Salud, 2012.

19. OLIVEIRA VJ, PENNA CMM. Discussing obstetric violence through the voices of women and health professionals. Texto contexto - enferm, 2017; 26(2): 1-10.

20. OMS. Organização Mundial de Saúde. Prevenção e eliminação de abusos, desrespeito e maus-tratos durante o parto em instituições de saúde. Genebra: Departamento de Saúde Reprodutiva e Pesquisa/OMS; 2014.

21. OMS. Organização Mundial de Saúde. Natureza, magnitude e consequências da violência sexual e da violência por parceiro íntimo. In: Prevenção da Violência Sexual e da Violência pelo Parceiro Íntimo Contra a Mulher: Ação e produção de evidência. Brasília: Organização Mundial de Saúde; 2012.

22. RODRIGUES FAC, et al. Violência obstétrica no processo de parturição em maternidades vinculadas à Rede Cegonha. Reprodução e Climatério, 2017; 32(2): 78-84.

23. RODRIGUES DP, et al. Intimate Partner Violence Against Pregnant Women: Study About The Repercussions On The Obstetric And Neonatal Results. Rev. esc. enferm, 2014; 48(2): 206-212.

24. RODRIGUES DP, et al. The pilgrimage in reproductive period: a violence in the field of obstetrics. Esc Anna Nery. 2015; 19(4): 614-620.

25. SILVA MG, et al. Violência obstétrica na visão de enfermeiras obstetras. Rev Rene, 2014;15(4): 720-728.

26. SILVA TM, et al. Violência obstétrica: a abordagem da temática na formação de enfermeiros obstétricos. Acta Paul Enferm. 2020; 33(1):1-8.

27. SOUZA MT, et al. Revisão integrativa: o que é e como fazer. Einstein, 2010, 8(1): 102-108.

28. URSI ES. Prevenção de lesões de pele no perioperatório: revisão integrativa da literatura. [dissertação]. Ribeirão Preto: Universidade de São Paulo, Escola de Enfermagem de Ribeirão Preto; 2005.

29. ZANARDO GLP, et al. Violência obstétrica no Brasil: uma revisão narrativa. Psicologia \& Sociedade, 2017; 29(1): 111. 